\title{
Towards an all-optical fingerprint of synthetic and natural microplastic fibers by Polarization-Sensitive Holographic Microscopy
}

Jaromír Běhal, ${ }^{1, \ddagger}$ Marika Valentino, $, 1,2, \ddagger$ Lisa Miccio, $,{ }^{*}, 1$ Vittorio Bianco, ${ }^{1}$ Simona Itri, ${ }^{1,3}$ Raffaella Mossotti, ${ }^{4}$ Giulia Dalla Fontana, ${ }^{4}$ Ettore Stella, ${ }^{5}$ Pietro Ferraro ${ }^{1}$

${ }^{1}$ Istituto di Scienze Applicate e Sistemi Intelligenti "Eduardo Caianiello" (ISASI-CNR), via Campi Flegrei 34, 80078 Pozzuoli, Napoli, Italy.

${ }^{2}$ Università degli Studi di Napoli Federico II, Dip. di Ingegneria Elettrica e delle Tecnologie dell'Informazione, via Claudio 21, 80125-Napoli, Italy.

${ }^{3}$ Department of Mathematics and Physics, University of Campania "L.Vanvitelli", 81100, Caserta, Italy. ${ }^{4}$ STIIMA-CNR Institute of Intelligent Industrial Technologies and Systems for Advanced Manufacturing National Research Council of Italy, C.so G. Pella 16, Biella, 13900, Italy.

${ }^{5}$ Istituto di Sistemi e Tecnologie Industriali Intelligenti per il Manifatturiero Avanzato (STIIMA-CNR), via Amendola 122 D/O, 70126 Bari (BA), Italy.

*Corresponding author: lisa.miccio@isasi.cnr.it

12 pages, 4 figures, 2 tables 


\section{Supporting Information 1: Pre-measurements}

The optical path itself may influence reconstructions provided by Eq. (9) of the main text. In such a case the Jones matrix of optical path $\boldsymbol{T}_{\boldsymbol{O P}}$ is superimposed on the sample's Jones matrix $\boldsymbol{T}_{\boldsymbol{S}}$, hence the reconstructed matrix $\boldsymbol{T}$ can be expressed in the form

$$
\boldsymbol{T}=\boldsymbol{T}_{O P} \boldsymbol{T}_{S}
$$

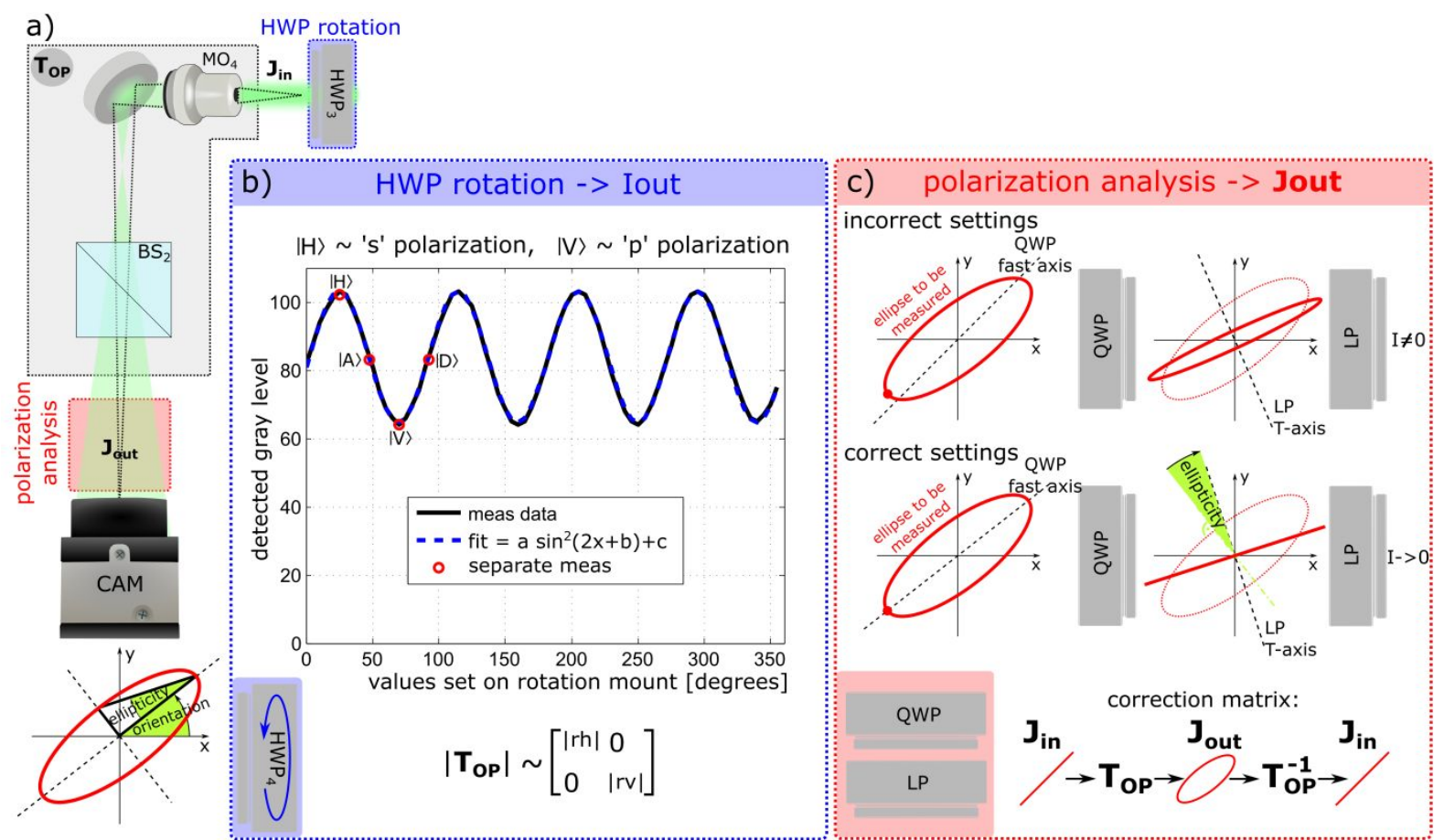

Figure S1. a) Sketch of the signal arm without sample. b) Periodic intensity changes due to polarization sensitive transmission of the optical path. Measured data, numerical fit, and separate measurements. Intensity minima and maxima correspond to input vertical and horizontal linear polarizations. c) Configuration, composed of quarter-wave plate $(Q W P)$ and linear polarizer $(L P)$, used to estimate parameters of output polarization ellipse.

Firstly, to test the possible polarization-dependent transmission of the signal arm (Figure $\mathrm{S} 1 \mathrm{a})$, the input linear polarization orientation was varied, rotating the half-wave plate $\mathrm{HWP}_{3}$. It was found that measured gray-level values exhibit periodical changes (Figure S1b), as may be expected in configurations with reflective elements present in the optical train ${ }^{1}$. It was also observed that the intensity maxima and minima correspond to the input horizontal and vertical linear polarization states, respectively. In addition, these polarizations remained unchanged in the output of the optical path, indicating their similarity with the eigenmodes. Because of this observation, the detected gray-level values were fitted by the function

$$
I=a \sin ^{2}(2 x+b)+c,
$$

corresponding to non-ideal linear polarizer ${ }^{2}$ with the parameters estimated as $a=38.3 \pm 0.2$, $b=40.7 \pm 0.1, c=64.6 \pm 0.1$. As a result, it was found that the ratio of absolute values of the vertical-polarization and the horizontal-polarization reflection coefficients is $\sqrt{I_{\min } / I_{\max }}$ $\approx 0.792$.

Polarization sensitive absorption of the optical path indicates that the output polarization states may consequently change. Hence, another measurement was performed in the proximity of the camera. Following the principles of the null-intensity compensation methods ${ }^{3}$, the configuration consisting of a quarter wave plate (QWP) and a linear polarizer (LP) was used to find parameters of the investigated polarization ellipse (Figure S1c). At the beginning of the measurement, the LP was inserted before the camera and set to find the intensity minimum. The 
intensity-minimum position corresponds to the orientation of a minor axis to which the major axis is perpendicular. Then, the quarter wave plate was placed before the linear polarizer. The fast axis of QWP was adjusted close to the expected major axis orientation, and an intensity minimum was found rotating the linear polarizer. This process was repeated for slightly different orientations of QWP until the sharp intensity extinction was found. Such a situation corresponds to the fact that the fast axis of QWP matches the major axis of the investigated polarization ellipse and compensates for its ellipticity. Hence linear polarization appears behind QWP. The extinct intensity minimum is then found rotating the polarizer perpendicularly to the linear polarization state. The difference between angles set on the linear polarizer before and after inserting the QWP determines the ellipticity of the measured polarization ellipse. The sense of the angle difference establishes the handedness of the ellipse. In this way, parameters of output polarization ellipse for diagonal initial polarization were found as $\psi=38^{\circ}, \chi=-7$ $\circ$. Similarly, the output polarization ellipse parameters $\psi=38^{\circ}, \chi=7^{\circ}$ were measured for an antidiagonal input polarization state. Both parameters with accuracy $\pm 2^{\circ}$. The Jones matrix of the optical setup was then estimated as

$$
\boldsymbol{T}_{\boldsymbol{O P}}=\left[\begin{array}{ll}
(1.00 \pm 0.02)+i(0.00 \pm 0.00) & (0.00 \pm 0.02)+i(0.00 \pm 0.00) \\
(0.00 \pm 0.03)+i(0.00 \pm 0.04) & (0.77 \pm 0.03)+i(-0.20 \pm 0.04)
\end{array}\right]
$$

and the corresponding inverse matrix as

$$
\boldsymbol{T}_{\boldsymbol{O P}}{ }^{-1}=\left[\begin{array}{ll}
(1.00 \pm 0.02)+i(0.00 \pm 0.00) & (0.00 \pm 0.02)+i(0.00 \pm 0.01) \\
(0.00 \pm 0.03)+i(0.00 \pm 0.05) & (1.22 \pm 0.04)+i(0.31 \pm 0.06)
\end{array}\right]
$$

Indeed, it can be noticed that the absolute value of the last $\boldsymbol{T}_{\boldsymbol{O P}}$ element $\left(\left|\boldsymbol{T}_{\boldsymbol{O P}, y y}\right| \approx 0.796\right)$ corresponds to the previously calculated absolute value of the reflection-coefficients ratio $\sqrt{I_{\min } / I_{\max }} \approx 0.792$.

Finally, to test the validity of the presented approach, a half-wave plate (HWP) with a fast axis set parallel to the horizontal direction was used as a known specimen. Based on the holographic complex-amplitude reconstruction approach, the interference record containing the multiplexed information about the horizontal and vertical polarization components was firstly Fourier transformed, the proper diffraction order selected, spatial carrier frequency removed, and inverse Fourier transformed. The restored complex amplitude was then corrected by the equally processed reference hologram ${ }^{4}$. In order to compensate for the potential defects caused by the temporal instability of the interferometric configuration, the constant phase, assessed inside the region without the sample, was deduced from the global phase map. Both polarization components of the Jones vector, horizontal and vertical, were treated identically, thus retrieved after the identical procedure. All the numerical process was applied for two snapshots recorded under diagonally and antidiagonally polarized illumination. Thus, Jones vectors $\boldsymbol{J}_{\text {OUT }}^{\boldsymbol{D}}, \boldsymbol{J}_{\text {OUT }}^{\boldsymbol{A}}$ were reconstructed, respectively, and corrected by the Jones matrix $\boldsymbol{T}_{\boldsymbol{O P}}{ }^{-1}$ to represent the correct states of polarization in the object space. Finally, the sample's Jones matrix $\boldsymbol{T}_{\boldsymbol{S}}$ was achieved as a consequence of Eq. (S1) and illustrated in Figure S2.

An ideal HWP is a transparent anisotropic optical element introducing a half-wave phase delay between ordinary and extraordinary polarization components. When the fast axis of HWP is set horizontal, amplitudes of diagonal Jones-matrix elements are equal to one, and amplitudes of the remaining elements are zero. Phases of the first and the last diagonal elements are $\pi / 2$ and $-\pi / 2$, respectively; hence the theoretically induced phase difference is $-\pi$. Values calculated inside the green dotted rectangles in Figure S2 are compared with the theoretical values in Table S1, approving good accuracy of the proposed measurements and numerical reconstructions with theory. The evaluated area is set to avoid border-regions of HWP. 
a) $\left|T s_{x x}\right|$

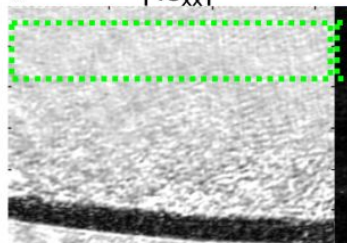

$\left|\mathbf{T s}_{\mathrm{vx}}\right|$

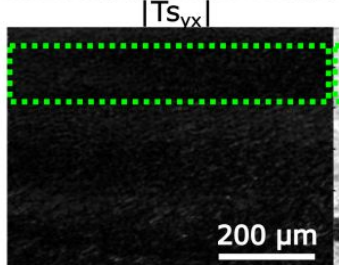

$\left|T s_{x y}\right|$

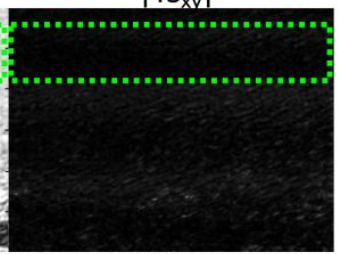

$\left|\mathrm{Ts}_{\mathrm{yy}}\right|$

b)

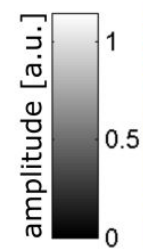

0.5

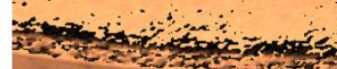

phase $\left(T s_{y x}\right)$

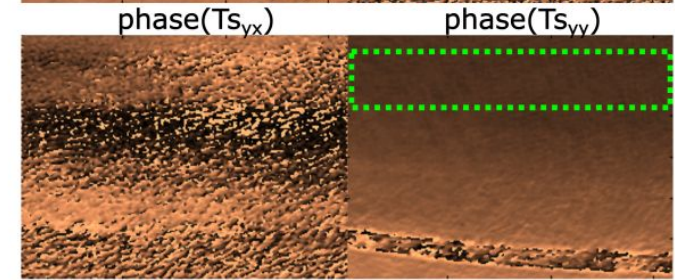

Figure S2. Reconstructed Jones matrix of half-wave plate with fast axis set parallel to the horizontal direction. Size of the green dotted rectangle is $\approx 130 \times 640 \mu \mathrm{m}^{2}$. a) Amplitude maps. b) Phase maps.

\begin{tabular}{|c|l|l|l|l|l|l|}
\hline HWP $\left(0^{\circ}\right)$ & & \multicolumn{1}{c|}{$\mathbf{T}_{\mathbf{S x x}}$} & \multicolumn{1}{c|}{$\mathbf{T}_{\mathbf{S x v}}$} & \multicolumn{1}{c|}{$\mathbf{T}_{\mathbf{S v x}}$} & \multicolumn{1}{c|}{$\mathbf{T}_{\mathbf{S v v}}$} & $\mathbf{T}_{\mathbf{S v v}} / \mathbf{T}_{\mathbf{S x x}}$ \\
\hline \multirow{2}{*}{$\begin{array}{c}\text { amplitude } \\
\text { [a.u.] }\end{array}$} & theory & 1.00 & 0.00 & 0.00 & 1.00 & 1.00 \\
\cline { 2 - 7 } & exper. & $0.99 \pm 0.04$ & $0.05 \pm 0.03$ & $0.06 \pm 0.03$ & $1.00 \pm 0.05$ & $1.01 \pm 0.05$ \\
\hline \multirow{2}{*}{ phase [radians] } & theory & 1.57 & ----- & ---- & -1.57 & -3.14 \\
\cline { 2 - 7 } & exper. & $1.59 \pm 0.06$ & ----- & ---- & $-1.59 \pm 0.07$ & $-3.18 \pm 0.05$ \\
\hline
\end{tabular}

Table S1. Comparison of the amplitude and the phase distributions calculated inside the green dotted rectangles (Figure S2) of size $\approx 130 \times 640 \mu \mathrm{m}^{2}$ with theory. 
Supporting Information 2: Features calculated for PA6, PET, PP, PA6.6, Cotton, Wool

PA6

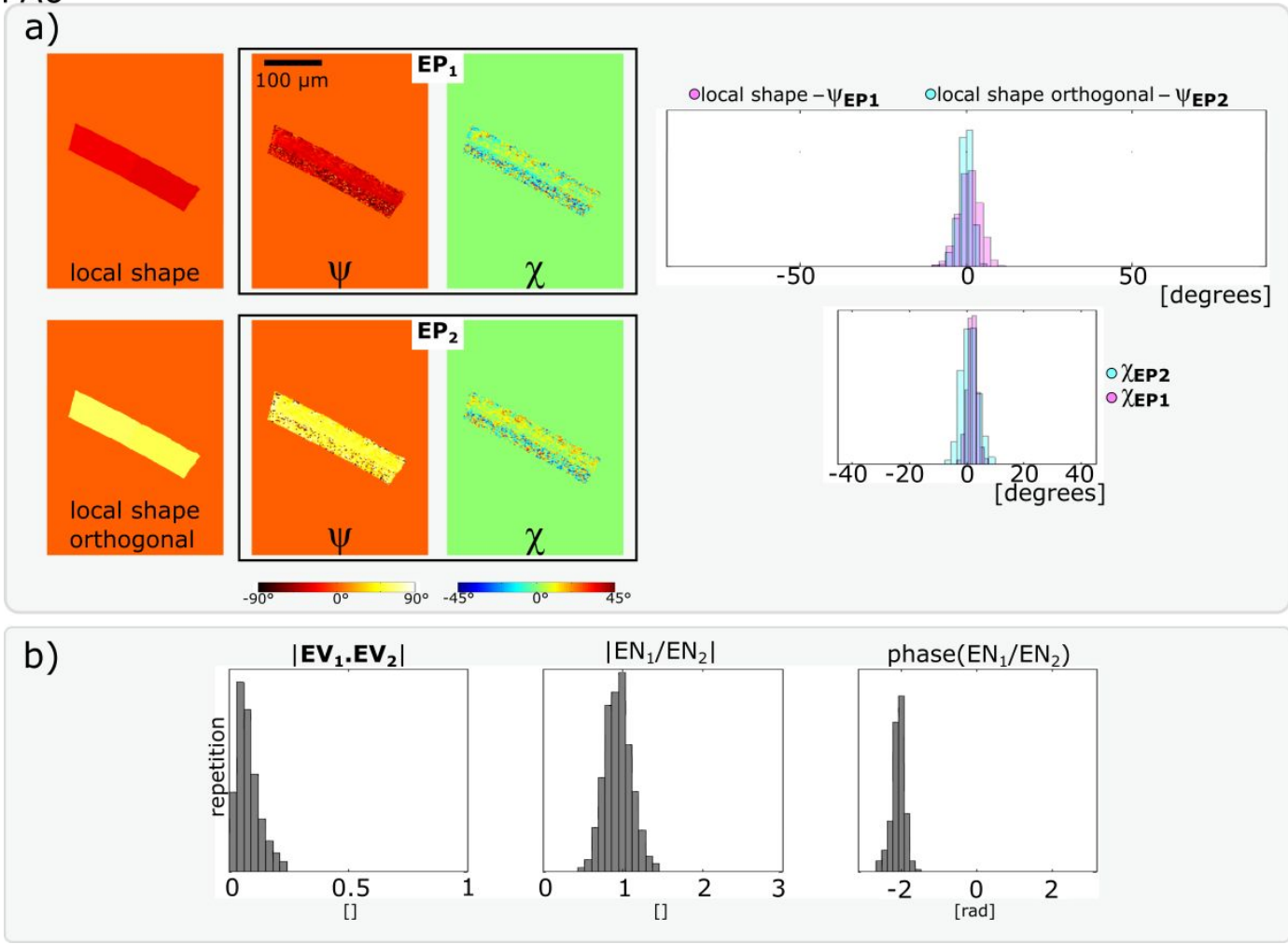

PET

a)

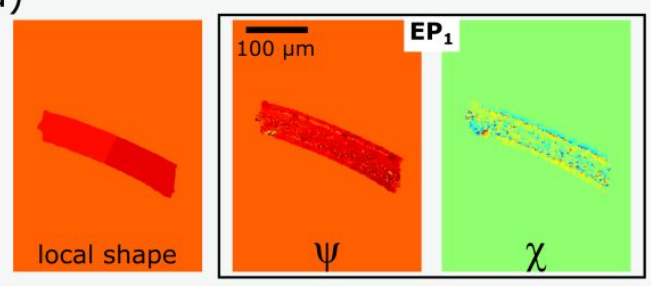

olocal shape - $\psi_{\text {EP1 }} \quad$ olocal shape orthogonal- $\psi_{\text {EP2 }}$
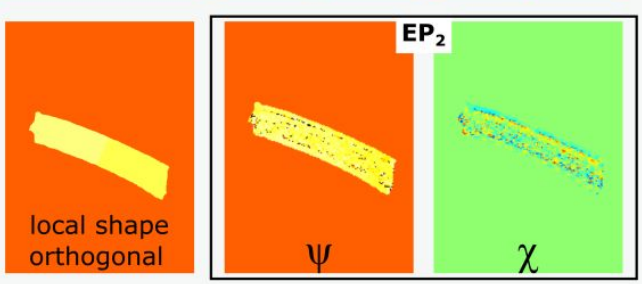

[degrees]

orthogonal

$\begin{array}{rrrrrr}-90^{\circ} & 0^{\circ} & 90^{\circ} & -45^{\circ} & 0^{\circ} & 45^{\circ}\end{array}$

b)
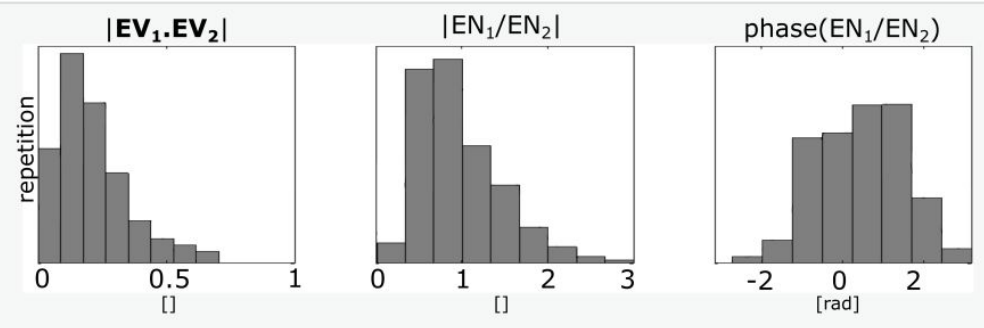
PP

a)
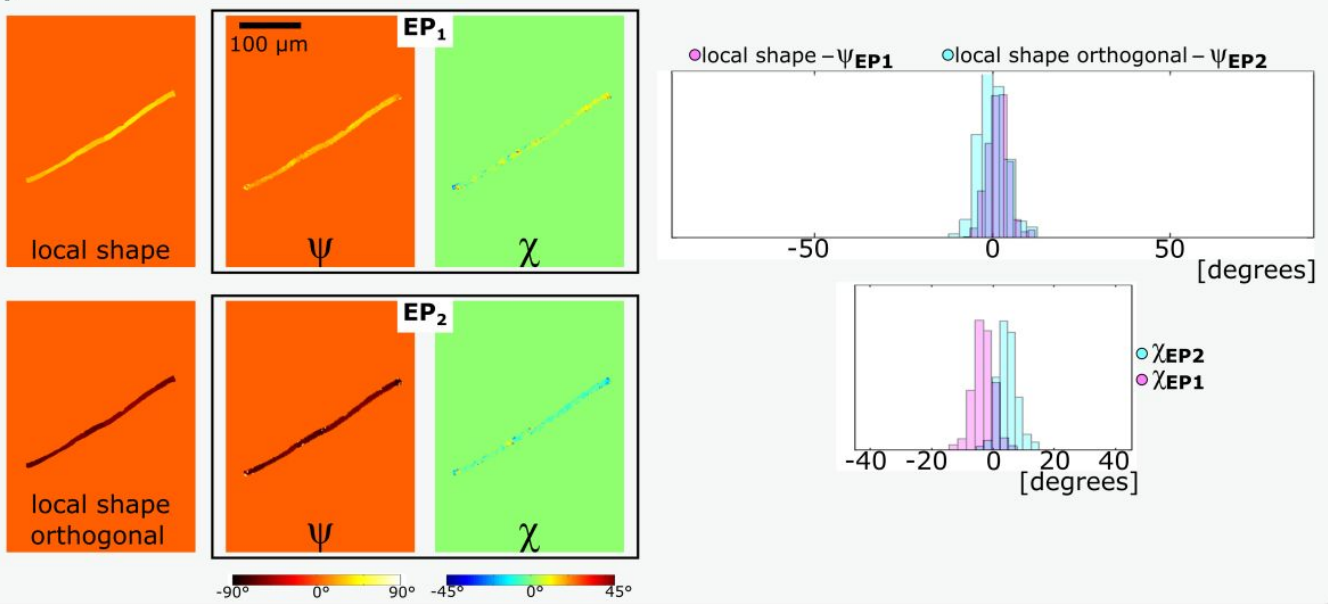

b)
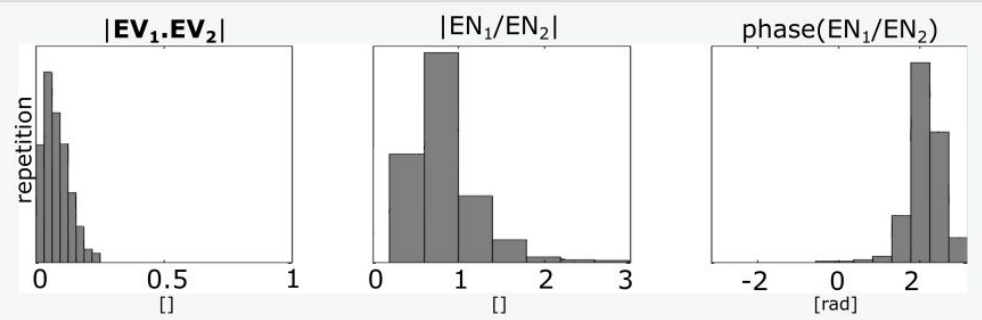

PA6.6

a)

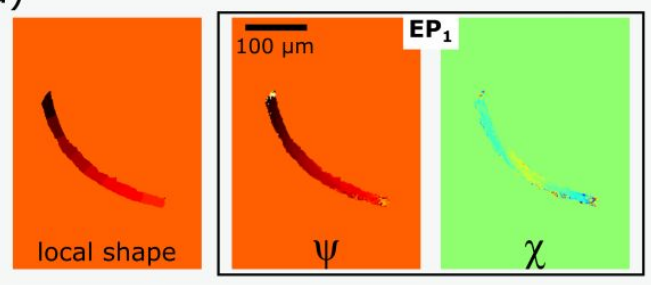

olocal shape $-\psi_{\text {EP1 }} \quad$ olocal shape orthogonal- $\psi_{\text {EP2 }}$
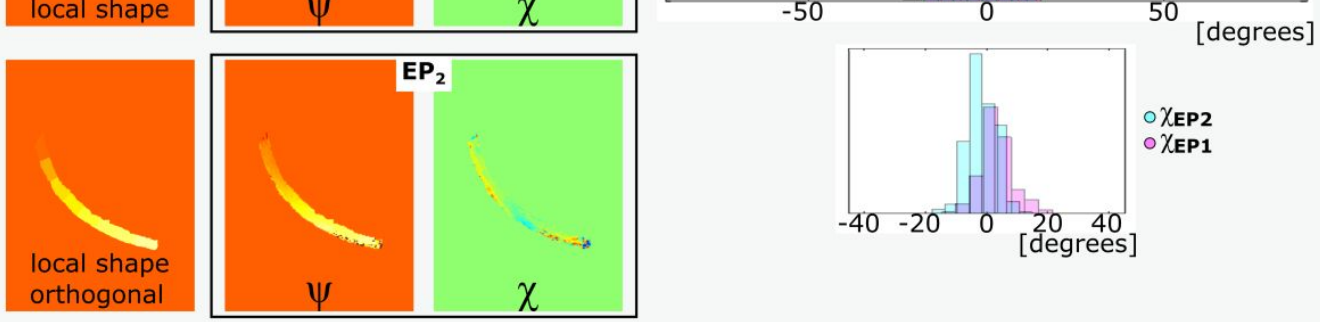

b)
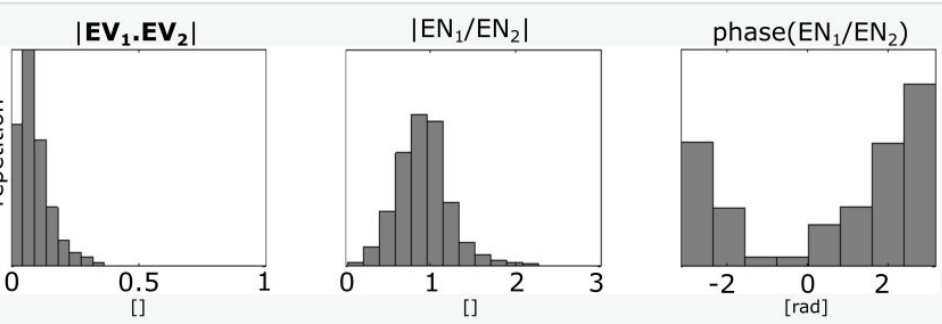


\section{Cotton}

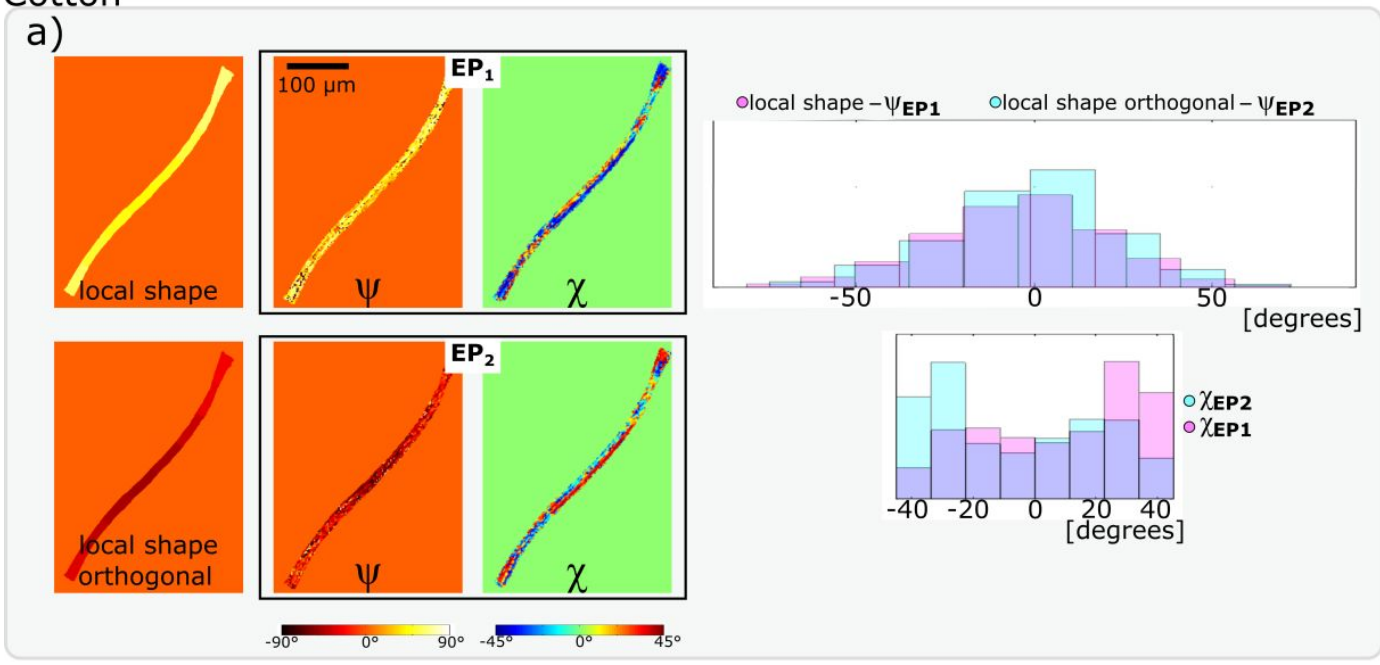

b)
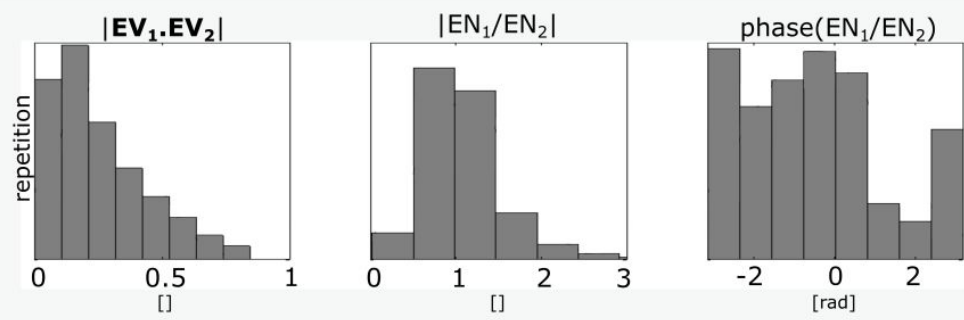

\section{Wool}

a)
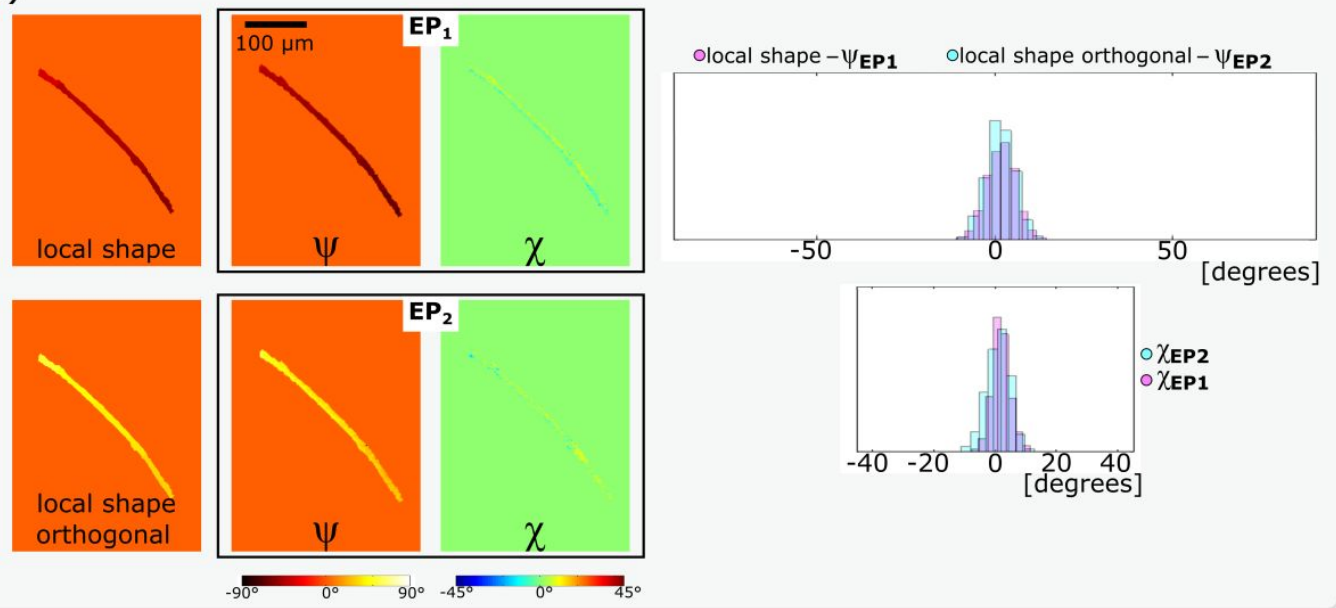

b)
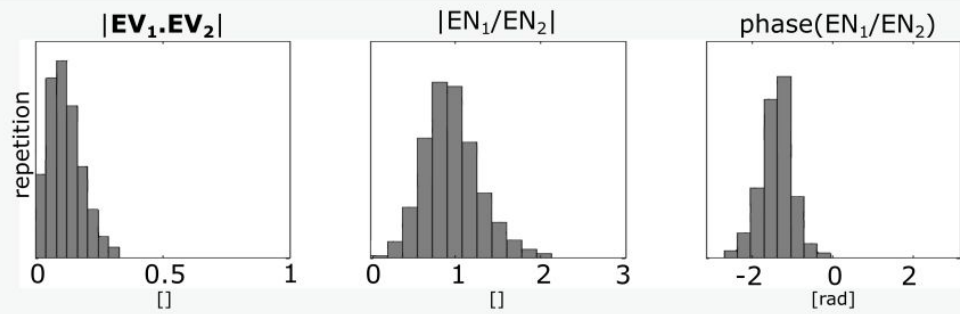

Figure S3 . Features of selected PA6, PET, PP, PA6.6, Cotton, and Wool MFFs, respectively. (a) Local shape of $M F$, direction orthogonal to the local shape, and parameters of $\boldsymbol{E P}_{\mathbf{1}}$ and $\boldsymbol{E P}_{\mathbf{2}}$ (major axis orientation $\psi$, ellipticity angle $\chi$ ). Moreover, the first histogram represents $\psi_{E P 1}$ relative to local shape, and $\psi_{E P 2}$ relative to the local shape orthogonal, respectively. Second histogram visualizes $\chi_{E P 1}$ and $\chi_{E P 2}$. (b) absolute value of inner product $\boldsymbol{E} \boldsymbol{V}_{\mathbf{1}} \cdot \boldsymbol{E} \boldsymbol{V}_{2}$, absolute value of $E N s^{\prime}$ ratio $E N_{1} / E N_{2}$, and phase of $E N_{1} / E N_{2}$. 
Supporting Information 3: Table of features and distribution characteristics

\begin{tabular}{|c|c|c|c|c|c|c|c|c|c|c|c|c|c|c|c|c|c|c|}
\hline \multirow[t]{2}{*}{ mean } & \multicolumn{2}{|c|}{$\left|E V_{1} \cdot E V_{2}\right|$} & \multicolumn{2}{|c|}{$\left|E N_{1} / E N_{2}\right|$} & \multicolumn{2}{|c|}{$\begin{array}{c}\text { phase } \\
\left(E N_{1} / E N_{2}\right)\end{array}$} & \multicolumn{2}{|c|}{$\chi_{E P 1}$} & \multicolumn{2}{|c|}{$\chi_{E P 2}$} & \multicolumn{2}{|c|}{$\begin{array}{c}\text { local shape - } \\
\psi_{E P 1}\end{array}$} & \multicolumn{2}{|c|}{$\begin{array}{l}\text { local shape orth. } \\
\qquad-\psi_{E P 2}\end{array}$} & $\begin{array}{r}\mathrm{re} \\
\left(E N_{1} /\right.\end{array}$ & & $\begin{array}{r}\text { in } \\
E N_{1}\end{array}$ & $\left.N_{2}\right)$ \\
\hline & mean & std & mean & std & mean & std & mean & std & mean & std & mean & std & mean & std & mean & std & mean & std \\
\hline PA6 & 0.08 & 0.03 & 0.92 & 0.07 & -0.57 & 1.36 & -0.10 & 1.44 & 0.51 & 1.39 & 0.48 & 1.78 & 0.62 & 1.69 & -0.37 & 0.43 & -0.11 & 0.45 \\
\hline PET & 0.17 & 0.04 & 0.72 & 0.13 & -0.18 & 0.50 & 0.12 & 1.49 & 0.59 & 1.55 & -0.09 & 2.42 & 1.36 & 2.40 & 0.17 & 0.16 & -0.03 & 0.19 \\
\hline PP & 0.09 & 0.02 & 0.89 & 0.16 & 1.49 & 0.50 & 0.08 & 2.17 & 0.56 & 2.10 & 0.49 & 0.82 & 1.05 & 1.14 & -0.31 & 0.21 & 0.48 & 0.15 \\
\hline PA6.6 & 0.18 & 0.06 & 0.90 & 0.07 & 0.51 & 0.54 & 0.92 & 4.94 & -0.44 & 4.74 & 0.49 & 3.37 & 1.28 & 3.89 & 0.26 & 0.31 & 0.25 & 0.28 \\
\hline Cott. & 0.18 & 0.04 & 1.01 & 0.09 & -0.40 & 0.51 & -2.87 & 10.03 & 3.46 & 9.99 & 0.10 & 2.30 & -0.10 & 3.42 & -0.05 & 0.24 & -0.22 & 0.26 \\
\hline Wool & 0.10 & 0.02 & 1.01 & 0.08 & -1.39 & 0.28 & 0.42 & 1.35 & 0.54 & 1.21 & 0.55 & 0.90 & 0.80 & 0.95 & 0.16 & 0.25 & -0.88 & 0.08 \\
\hline$\frac{\text { media }}{\underline{\mathrm{n}}}$ & $\left.\right|^{E V_{1}}$ & & $1^{E N_{1}}$ & & $\begin{array}{c}\mathrm{ph}_{\mathrm{i}} \\
\left(E N_{1}\right.\end{array}$ & & & & & & $\begin{array}{r}\text { local s } \\
\psi_{E}\end{array}$ & & $\begin{array}{r}\text { local sha } \\
-\psi_{t}\end{array}$ & orth. & $\begin{array}{r}\mathrm{re} \\
\left(E N_{1} /\right.\end{array}$ & & $\begin{array}{r}\text { in } \\
E N_{1}\end{array}$ & \\
\hline & mean & std & mean & std & mean & std & mean & std & mean & std & mean & std & mean & std & mean & std & mean & std \\
\hline PA6 & 0.06 & 0.02 & 0.89 & 0.05 & -0.80 & 1.78 & 0.00 & 1.47 & 0.39 & 1.44 & 0.48 & 1.79 & 0.64 & 1.77 & -0.39 & 0.45 & -0.14 & 0.46 \\
\hline PET & 0.14 & 0.03 & 0.66 & 0.11 & -0.30 & 0.65 & 0.08 & 1.60 & 0.62 & 1.51 & -0.07 & 2.37 & 1.41 & 2.23 & 0.20 & 0.20 & -0.07 & 0.22 \\
\hline PP & 0.08 & 0.01 & 0.79 & 0.09 & 1.94 & 0.28 & 0.03 & 2.06 & 0.51 & 2.07 & 0.48 & 0.70 & 1.00 & 1.08 & -0.35 & 0.18 & 0.48 & 0.13 \\
\hline PA6.6 & 0.14 & 0.05 & 0.88 & 0.05 & 0.65 & 0.64 & 0.90 & 5.09 & -0.62 & 5.10 & 0.48 & 2.37 & 1.13 & 2.90 & 0.33 & 0.35 & 0.31 & 0.32 \\
\hline Cott. & 0.15 & 0.04 & 0.92 & 0.06 & -0.61 & 0.83 & -3.39 & 14.71 & 4.22 & 14.49 & 0.54 & 1.94 & 0.31 & 3.36 & -0.08 & 0.29 & -0.25 & 0.31 \\
\hline Wool & 0.09 & 0.02 & 0.96 & 0.05 & -1.37 & 0.26 & 0.49 & 1.40 & 0.52 & 1.23 & 0.59 & 0.82 & 0.84 & 0.89 & 0.16 & 0.24 & -0.85 & 0.07 \\
\hline mode & $\left.\right|^{E V_{1}}$ & $V_{2}$ & $\left.\right|^{E N_{1}}$ & & $\begin{array}{r}\text { ph } \\
\left(E N_{1}\right)\end{array}$ & & & & & & $\begin{array}{r}\text { local s } \\
\psi_{E}\end{array}$ & & $\begin{array}{r}\text { local sha } \\
-\psi_{1}\end{array}$ & & $\begin{array}{r}\mathrm{re} \\
\left(E N_{1}\right.\end{array}$ & & $\begin{array}{r}\text { in } \\
E N_{1}\end{array}$ & \\
\hline & mean & std & mean & std & mean & std & mean & std & mean & std & mean & std & mean & std & mean & std & mean & std \\
\hline PA6 & 0.04 & 0.01 & 0.87 & 0.08 & -1.19 & 1.95 & -0.23 & 2.06 & 0.62 & 1.70 & 0.46 & 2.18 & 0.72 & 2.32 & -0.39 & 0.52 & -0.27 & 0.53 \\
\hline PET & 0.09 & 0.02 & 0.60 & 0.12 & -0.41 & 1.17 & 0.20 & 1.99 & 0.76 & 1.61 & -0.22 & 2.74 & 1.83 & 2.56 & 0.24 & 0.30 & -0.18 & 0.30 \\
\hline PP & 0.06 & 0.01 & 0.72 & 0.09 & 2.14 & 0.39 & 0.02 & 2.25 & 0.33 & 2.46 & 0.30 & 1.28 & 0.75 & 1.43 & -0.35 & 0.25 & 0.49 & 0.13 \\
\hline PA6.6 & 0.08 & 0.03 & 0.87 & 0.08 & 0.59 & 1.00 & 0.91 & 4.90 & -0.87 & 5.75 & 0.35 & 2.74 & 0.88 & 3.05 & 0.51 & 0.41 & 0.30 & 0.47 \\
\hline Cott. & 0.08 & 0.02 & 0.83 & 0.08 & -0.89 & 1.68 & -4.19 & 21.05 & 3.98 & 20.89 & 0.77 & 3.77 & 0.80 & 3.34 & -0.09 & 0.45 & -0.37 & 0.47 \\
\hline Wool & 0.07 & 0.02 & 0.89 & 0.07 & -1.36 & 0.26 & 0.67 & 1.67 & 0.40 & 1.44 & 0.73 & 1.13 & 0.95 & 1.24 & 0.16 & 0.24 & -0.84 & 0.09 \\
\hline$\frac{\text { mean }}{\underline{\mathrm{AD}}}$ & ${ }^{E V_{1}}$ & $V_{2}$ & $E N_{1}$ & & $\begin{array}{r}\text { ph } \\
\left(E N_{1}\right)\end{array}$ & & & & & & $\begin{array}{r}\text { local s } \\
\psi_{E}\end{array}$ & & $\begin{array}{r}\text { local sha } \\
-\psi_{t}\end{array}$ & & $\begin{array}{r}\mathrm{re} \\
\left(E N_{1}\right.\end{array}$ & & $\begin{array}{r}\text { in } \\
\left(E N_{1}\right.\end{array}$ & \\
\hline & mean & std & mean & std & mean & std & mean & std & mean & std & mean & std & mean & std & mean & std & mean & std \\
\hline PA6 & 0.04 & 0.03 & 0.19 & 0.05 & 0.63 & 0.51 & 3.43 & 2.55 & 3.36 & 2.50 & 2.75 & 1.82 & 2.49 & 1.24 & 0.32 & 0.17 & 0.33 & 0.16 \\
\hline PET & 0.09 & 0.02 & 0.27 & 0.07 & 1.08 & 0.24 & 4.78 & 1.25 & 4.42 & 1.07 & 5.64 & 1.57 & 5.85 & 1.66 & 0.39 & 0.10 & 0.41 & 0.09 \\
\hline PP & 0.05 & 0.01 & 0.34 & 0.11 & 0.66 & 0.19 & 2.85 & 0.59 & 2.94 & 0.63 & 3.33 & 0.62 & 3.31 & 0.69 & 0.40 & 0.11 & 0.37 & 0.10 \\
\hline PA6.6 & 0.10 & 0.04 & 0.21 & 0.05 & 0.85 & 0.31 & 7.77 & 3.25 & 7.85 & 3.27 & 7.37 & 2.49 & 7.48 & 2.63 & 0.41 & 0.14 & 0.44 & 0.12 \\
\hline Cott. & 0.10 & 0.02 & 0.37 & 0.08 & 1.21 & 0.35 & 15.2 & 6.40 & 15.1 & 6.39 & 11.4 & 4.71 & 11.6 & 4.67 & 0.58 & 0.11 & 0.56 & 0.10 \\
\hline Wool & 0.05 & 0.01 & 0.26 & 0.07 & 0.30 & 0.08 & 2.71 & 0.53 & 2.56 & 0.53 & 3.01 & 0.61 & 3.19 & 0.67 & 0.27 & 0.08 & 0.25 & 0.06 \\
\hline$\frac{\text { media }}{\underline{\text { nAD }}}$ & $\left.\right|^{E V_{1}}$ & $V_{2}$ & $\left.\right|^{E N_{1}}$ & & $\begin{array}{c}\text { ph } \\
\left(E N_{1}\right.\end{array}$ & & & & & & $\begin{array}{r}\text { local s } \\
\psi_{E}\end{array}$ & & $\begin{array}{r}\text { local sha } \\
-\psi_{t}\end{array}$ & & $\begin{array}{r}\mathrm{re} \\
\left(E N_{1}\right.\end{array}$ & & $\begin{array}{r}\text { in } \\
E N_{1}\end{array}$ & \\
\hline & mean & std & mean & std & mean & std & mean & std & mean & std & mean & std & mean & std & mean & std & mean & std \\
\hline PA6 & 0.03 & 0.01 & 0.15 & 0.04 & 0.45 & 0.36 & 2.17 & 1.05 & 2.08 & 0.96 & 1.85 & 0.78 & 1.87 & 0.76 & 0.24 & 0.12 & 0.28 & 0.15 \\
\hline PET & 0.06 & 0.02 & 0.20 & 0.04 & 0.89 & 0.23 & 3.49 & 0.96 & 3.18 & 0.80 & 4.24 & 1.44 & 4.24 & 1.20 & 0.31 & 0.09 & 0.32 & 0.09 \\
\hline PP & 0.04 & 0.01 & 0.23 & 0.05 & 0.50 & 0.17 & 2.22 & 0.44 & 2.24 & 0.41 & 2.61 & 0.46 & 2.58 & 0.56 & 0.30 & 0.08 & 0.26 & 0.06 \\
\hline PA6.6 & 0.07 & 0.03 & 0.16 & 0.03 & 0.71 & 0.35 & 5.13 & 2.08 & 5.12 & 2.11 & 5.07 & 1.55 & 5.21 & 1.67 & 0.32 & 0.13 & 0.35 & 0.12 \\
\hline Cott. & 0.07 & 0.02 & 0.25 & 0.04 & 1.00 & 0.38 & 11.1 & 6.41 & 11.0 & 6.39 & 8.39 & 4.01 & 8.44 & 4.02 & 0.46 & 0.11 & 0.44 & 0.10 \\
\hline Wool & 0.04 & 0.01 & 0.19 & 0.04 & 0.23 & 0.06 & 2.20 & 0.43 & 2.07 & 0.43 & 2.47 & 0.51 & 2.65 & 0.57 & 0.21 & 0.05 & 0.19 & 0.04 \\
\hline$\underline{\text { STD }}$ & $\left.\right|^{E V_{1}}$ & $V_{2}$ & $\left.\right|^{E N_{1}}$ & & $\begin{array}{c}\text { ph } \\
\left(E N_{1}\right.\end{array}$ & & & & & & $\begin{array}{r}\text { local s } \\
\psi_{E}\end{array}$ & & $\begin{array}{r}\text { local sha } \\
-\psi_{t}\end{array}$ & & $\begin{array}{r}\mathrm{re} \\
\left(E N_{1}\right.\end{array}$ & & $\begin{array}{r}\text { in } \\
E N_{1}\end{array}$ & \\
\hline & mean & std & mean & std & mean & std & mean & std & mean & std & mean & std & mean & std & mean & std & mean & std \\
\hline PA6 & 0.06 & 0.04 & 0.24 & 0.06 & 0.76 & 0.58 & 4.66 & 3.74 & 4.58 & 3.69 & 3.93 & 2.93 & 3.94 & 2.88 & 0.40 & 0.20 & 0.41 & 0.18 \\
\hline PET & 0.12 & 0.02 & 0.36 & 0.12 & 1.34 & 0.25 & 6.45 & 1.65 & 6.02 & 1.46 & 7.65 & 1.99 & 7.87 & 2.18 & 0.50 & 0.13 & 0.53 & 0.12 \\
\hline PP & 0.06 & 0.01 & 0.48 & 0.19 & 0.86 & 0.22 & 3.72 & 0.78 & 3.85 & 0.83 & 4.38 & 0.86 & 4.37 & 0.97 & 0.55 & 0.16 & 0.52 & 0.17 \\
\hline PA6.6 & 0.13 & 0.04 & 0.30 & 0.08 & 1.08 & 0.38 & 10.69 & 4.47 & 10.82 & 4.40 & 10.18 & 3.54 & 10.26 & 3.69 & 0.51 & 0.16 & 0.55 & 0.13 \\
\hline Cott. & 0.13 & 0.03 & 0.52 & 0.13 & 1.47 & 0.35 & 18.0 & 6.63 & 17.9 & 6.64 & 15.3 & 5.79 & 15.6 & 5.77 & 0.75 & 0.14 & 0.73 & 0.14 \\
\hline Wool & 0.06 & 0.01 & 0.35 & 0.11 & 0.40 & 0.11 & 3.46 & 0.68 & 3.27 & 0.69 & 3.88 & 0.85 & 4.05 & 0.87 & 0.35 & 0.09 & 0.34 & 0.10 \\
\hline$\frac{\text { skewn }}{\underline{\text { ess }}}$ & $\left.\right|^{E V_{1}}$ & $V_{2}$ & $\left.\right|^{E N_{1}}$ & & $\begin{array}{c}\text { ph } \\
\left(E N_{1}\right.\end{array}$ & & & & & & $\begin{array}{r}\text { local s } \\
\psi_{E}\end{array}$ & & $\begin{array}{r}\text { local sha } \\
-\psi_{t}\end{array}$ & orth. & $\begin{array}{r}\mathrm{re} \\
\left(E N_{1}\right.\end{array}$ & & $\begin{array}{r}\text { in } \\
\left(E N_{1}\right.\end{array}$ & \\
\hline & mean & std & mean & std & mean & std & mean & std & mean & std & mean & std & mean & std & mean & std & mean & std \\
\hline PA6 & 1.10 & 0.51 & 0.51 & 0.67 & -0.25 & 0.66 & 0.03 & 0.28 & 0.04 & 0.34 & -0.08 & 0.36 & 0.06 & 0.37 & -0.05 & 0.51 & 0.18 & 0.39 \\
\hline PET & 1.16 & 0.22 & 1.02 & 0.70 & 0.08 & 0.20 & -0.01 & 0.24 & -0.03 & 0.14 & -0.02 & 0.32 & $-\mathbf{- 0 . 0 3}$ & 0.42 & -0.32 & 0.32 & 0.21 & 0.30 \\
\hline PP & 1.17 & 0.23 & 1.53 & 1.06 & -0.55 & 0.33 & 0.06 & 0.26 & 0.06 & 0.24 & -0.01 & 0.21 & 0.01 & 0.22 & 0.06 & 0.36 & -0.04 & 0.31 \\
\hline PA6.6 & 1.21 & 0.29 & 0.74 & 0.79 & -0.18 & 0.31 & 0.03 & 0.30 & -0.05 & 0.34 & -0.14 & 0.41 & 0.05 & 0.43 & -0.43 & 0.46 & -0.26 & 0.38 \\
\hline Cott. & 1.17 & 0.19 & 1.78 & 0.83 & 0.15 & 0.38 & 0.19 & 0.68 & -0.21 & 0.67 & -0.08 & 0.17 & -0.11 & 0.16 & 0.06 & 0.27 & 0.15 & 0.23 \\
\hline Wool & 0.86 & 0.16 & 1.01 & 0.75 & -0.54 & 0.35 & -0.05 & 0.12 & 0.02 & 0.10 & 0.03 & 0.16 & -0.02 & 0.14 & 0.07 & 0.22 & -0.43 & 0.36 \\
\hline$\frac{\text { kurtos }}{\underline{\text { is }}}$ & $\left.\right|^{E V_{1}}$ & $V_{2}$ & $E N_{1}$ & & $\begin{array}{c}\text { ph } \\
\left(E N_{1}\right.\end{array}$ & & & & & & $\begin{array}{r}\text { local s } \\
\psi_{E}\end{array}$ & & $\begin{array}{r}\text { local sha } \\
-\psi_{L}\end{array}$ & & $\begin{array}{r}\mathrm{re} \\
\left(E N_{1}\right)\end{array}$ & & $\left(E N_{1}\right.$ & \\
\hline & mean & std & mean & std & mean & std & mean & std & mean & std & mean & std & mean & std & mean & std & mean & std \\
\hline PA6 & 4.15 & 1.44 & 3.45 & 0.71 & 3.30 & 1.39 & 3.59 & 0.97 & 3.59 & 0.83 & 4.15 & 1.99 & 3.81 & 1.32 & 3.07 & 0.89 & 2.88 & 0.57 \\
\hline PET & 4.09 & 0.71 & 5.37 & 3.41 & 2.72 & 0.50 & 4.22 & 0.55 & 4.33 & 0.54 & 4.62 & 0.92 & 4.52 & 0.97 & 3.76 & 1.07 & 3.42 & 0.72 \\
\hline PP & 4.35 & 0.73 & 7.30 & 4.46 & 4.23 & 1.03 & 3.73 & 0.48 & 3.77 & 0.49 & 3.97 & 0.75 & 3.91 & 0.67 & 4.88 & 1.86 & 5.64 & 2.92 \\
\hline PA6.6 & 4.08 & 1.09 & 5.54 & 3.40 & 2.92 & 0.80 & 4.00 & 1.02 & 3.96 & 0.94 & 4.73 & 1.05 & 4.69 & 0.92 & 3.33 & 0.99 & 3.14 & 1.11 \\
\hline Cott. & 3.93 & 0.58 & 8.29 & 3.37 & 2.73 & 0.95 & 2.61 & 0.96 & 2.64 & 0.98 & 4.18 & 0.83 & 4.22 & 0.86 & 4.22 & 1.25 & 4.39 & 1.34 \\
\hline Wool & 3.48 & 0.43 & 5.46 & 2.69 & 5.53 & 1.24 & 3.28 & 0.27 & 3.35 & 0.24 & 3.28 & 0.33 & 3.23 & 0.39 & 4.55 & 1.51 & 4.92 & 2.01 \\
\hline
\end{tabular}


Table S2. Mean, median, mode, mean absolute deviation, median absolute deviation, standard deviation, skewness, and kurtosis of the following features: (a) absolute value of inner product $\boldsymbol{E V}_{\mathbf{1}} \cdot \boldsymbol{E V}_{\mathbf{2}}$. (b) absolute value of the eigennumbers' ratio $E N_{1} / E N_{2}$. (c) phase of $E N_{1} / E N_{2}$. (d) ellipticity of $\boldsymbol{E P}_{\mathbf{1}}$. (e) ellipticity of $\boldsymbol{E} \boldsymbol{P}_{2}$. (f) major axis orientation of $\boldsymbol{E P}_{\mathbf{1}}$ relative to the local shape. $(g)$ major axis orientation of $\boldsymbol{E}_{\mathbf{2}}$ relative to the direction perpendicular to the local shape. (h) real part of $E N_{1} / E N_{2}$. (i) imaginary part of $E N_{1} / E N_{2}$. Here the little-font 'mean' stands for mean value of all distribution characteristics (represented by the bold square in Figure S4) and the little-font 'std' stands for standard deviation of all distribution characteristics (represented by the bold error bar in Figure S4), which were calculated for MFFs of the same class. 
Supporting Information 4: Graphical form of features and distribution characteristics

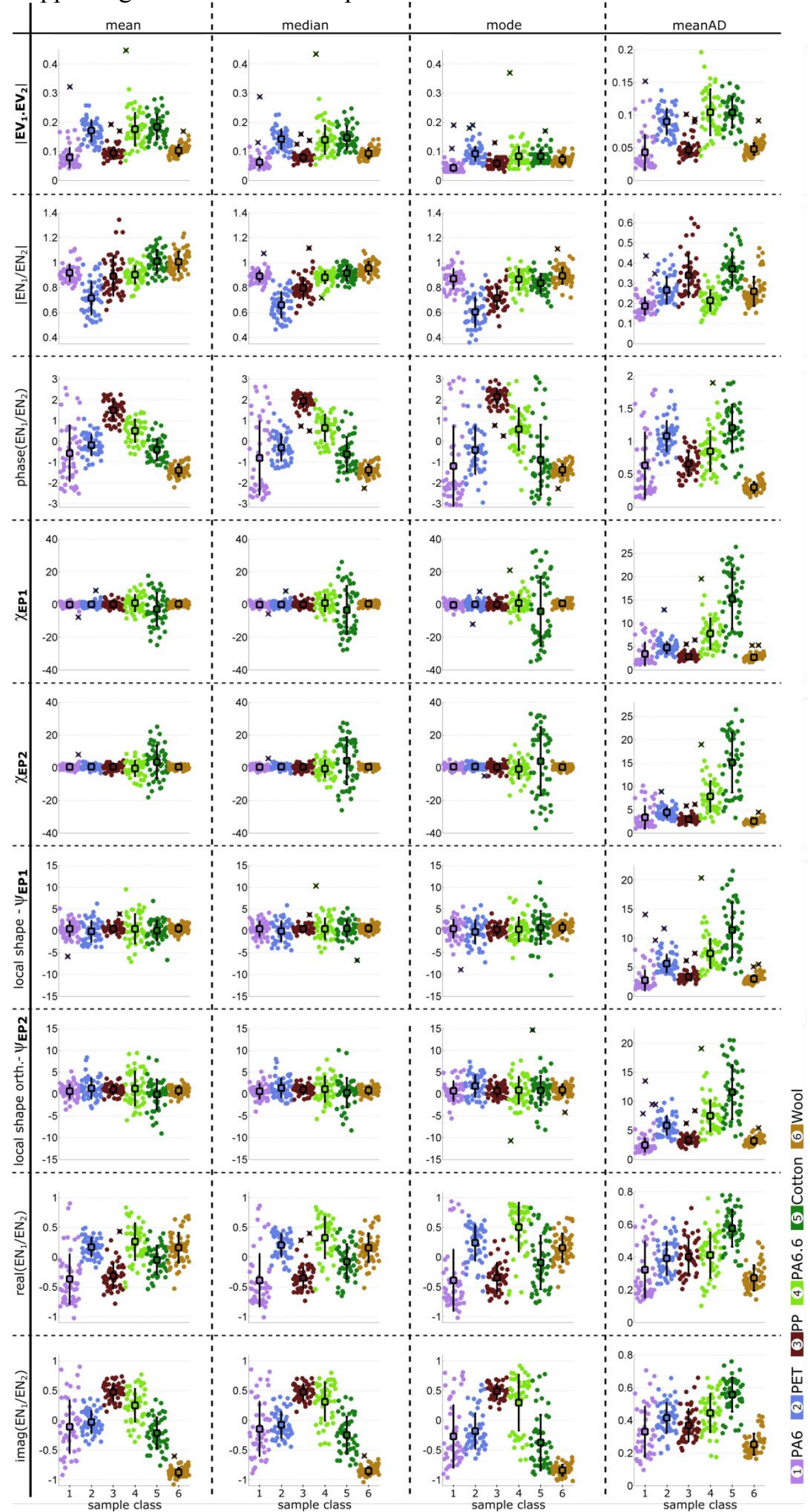




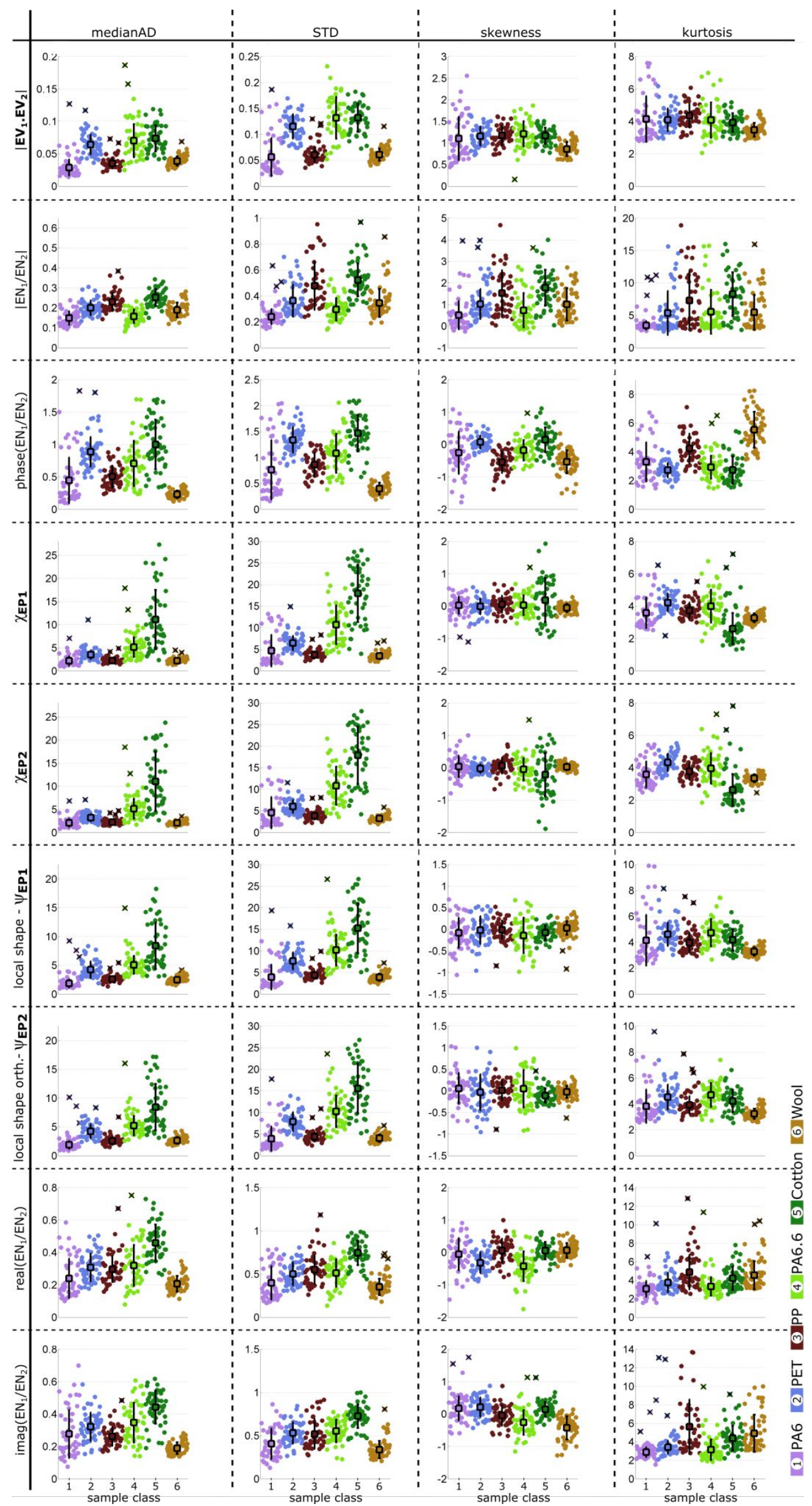

Figure S4. Mean, median, mode, mean absolute deviation, median absolute deviation, standard deviation, skewness, and kurtosis of the same features as are described in the caption of Table $S 2$. 
Supporting Information 5: Comparison of the selected distribution characteristics

Specifically, the distribution of the $\left|\boldsymbol{E} \boldsymbol{V}_{\mathbf{1}} \cdot \boldsymbol{E} \boldsymbol{V}_{\mathbf{2}}\right|$ appear moderately asymmetric as shown in SI - 2, hence mean value, median and mode differ (SI - 3). Despite this observation, all these characteristics tend to be higher for PET, PA6.6, and Cotton comparing PA6, PP, and Wool (SI - 3; Table S2 - yellow). Differences between mean value, median, and mode of the $\boldsymbol{E} \boldsymbol{P} s^{\prime}$ ellipticity in case of Cotton (SI - 3; Table S2 - blue) also indicate asymmetry of the distribution, as is illustrated in SI - 2 - Cotton a. Additionally, mode of $\boldsymbol{E} \boldsymbol{P} S^{\prime}$ ellipticity, seems to be a valuable parameter for distinguishing Cotton fibers, because its values are situated in peripheries of the range $\left\langle-45^{\circ}, 45^{\circ}\right\rangle$; hence practically avoid zero position (SI - 4). In opposite, even though PA6.6 exhibits a higher standard deviation of the $\boldsymbol{E} \boldsymbol{P} S^{\prime}$ ellipticity with respect to PA6, PET, PP, and Wool, the difference between mean value, median and mode is negligible (SI - 3; Table S2 - blue).

Moreover, meanAD, medianAD, and $\mathrm{STD}$ of the $\left|\boldsymbol{E} \boldsymbol{V}_{\mathbf{1}} \cdot \boldsymbol{E} \boldsymbol{V}_{\mathbf{2}}\right|$ are qualitatively comparable with distributions of its mean value, median and mode; thus, they may allow to distinguish higher-value PET, PA6.6, and Cotton relative to the lower-value PA6, PP, and Wool fibers, respectively (SI - 3; Table S2 - yellow). It was also observed that there are notable differences between meanAD and medianAD of the absolute value of the $E N s^{\prime}$ ratio in cases of PP and Cotton (SI - 3; Table S2 - purple). Additionally, it can be pointed out that MeanAD, medianAD, or STD of the $\boldsymbol{E} \boldsymbol{P} s^{\prime}$ ellipticity, seems to be a valuable parameter for distinguishing Cotton MFFs and the same is valid for orientation of the EPS (SI - 3; Table S2 - orange; SI 4), even though there is a certain overlap with PA6.6 fibers.

As well as the parameters mentioned above, also skewness and kurtosis provide certain separation of the fiber types, e.g., for phase of the ENs' ratio and $\boldsymbol{E} \boldsymbol{P} s^{\prime}$ ellipticity (SI - 4).

\section{REFERENCES}

(1) Born, M.; Wolf, E. Principles of Optics; Electromagnetic Theory of Propagation, Interference, and Diffraction of Light; Oxford: Pergamon Press, 1964.

(2) Goldstein Collett, Edward, D. H. Polarized Light.; Marcel Dekker: New York, 2003.

(3) Montarou, C. C.; Gaylord, T. K. Two-Wave-Plate Compensator Method for SinglePoint Retardation Measurements. Appl. Opt. 2004, 43 (36), 6580-6595. https://doi.org/10.1364/AO.43.006580.

(4) Ferraro, P.; Nicola, S. De; Finizio, A.; Coppola, G.; Grilli, S.; Magro, C.; Pierattini, G. Compensation of the Inherent Wave Front Curvature in Digital Holographic Coherent Microscopy for Quantitative Phase-Contrast Imaging. Appl. Opt. 2003, 42 (11), 1938 1946. https://doi.org/10.1364/AO.42.001938. 